\title{
Salivary Biomarkers of Nutritional Status: a Systematic Review
}

\section{Abstract \\ Introduction:}

Full nutritional assessments are currently complex and invasive. There is a need for a non-invasive, timely and cost-effective method to assess nutritional status. Evidence indicates the usefulness of saliva in diagnosing oral or systemic disorders. Saliva is suggested to be a reliable and non-invasive matrix in which to measure nutritional biomarkers. The aim of this work was to systematically review the evidence for salivary biomarkers as indicators of nutritional status.

\section{Materials and Methods:}

Studies identifying salivary biomarkers in relation to nutritional status or dietary intake outcomes were included. A search strategy combined terms "saliva" AND "biomarkers" AND "nutrition". Four databases were searched, MEDLINE, EMBASE, Web of Science and Scopus. All study designs conducted in humans of all ages, from all countries and settings were included. Non-English and animal studies were excluded. Risk of bias was assessed using the Newcastle-Ottawa Scale and Cochrane Risk of Bias tool where applicable. (PROSPERO Registration Number:CRD42018107667)

\section{Results:}

6585 papers were identified, 4836 papers remained after removing duplicates, 4715 were irrelevant, 134 full-texts were assessed for eligibility and 64 papers included in the final analysis. A number of potential salivary biomarkers related to nutritional status were identified including: total protein, albumin, prealbumin, transferrin, ferritin and iron. Total protein levels in saliva in malnourished individuals were significantly different to controls in $7 / 10$ studies $(70 \%)$. In one study conducted in individuals with iron deficiency anaemia (IDA), total protein was significantly different to controls. Albumin levels in malnourished individuals were significantly different to controls in $5 / 8$ studies $(62.5 \%)$. Prealbumin and transferrin levels in malnourished individuals were significantly different to controls in $3 / 3$ studies $(100 \%)$. In one study conducted in malnourished individuals, salivary ferritin levels was significantly different to controls. Ferritin levels in individuals with IDA were significantly different to controls in $3 / 3$ studies $(100 \%)$. Iron levels in individuals with IDA were significantly different in $2 / 2$ studies $(100 \%)$. However, even within the studies above where significant differences existed, the direction of salivary biomarker differences was sometimes inconsistent. For example, total protein in malnourished individuals was significantly lower than controls in three studies, higher in three studies and one showed mixed findings. In addition, overall the quality of evidence available was very poor.

\section{Discussion:}

Despite conflicting evidence in salivary nutritional biomarkers in individuals with malnutrition or IDA, saliva may be a useful noninvasive matrix to assess nutritional status. Further high quality research exploring the utility of these biomarkers is required.

\section{Conflict of Interest}

There is no conflict of interest. 